\title{
A SOLUTION METHOD OF THE MODIFIED BLACK-SCHOLES MODEL
}

\section{QIAN SUN and LINGYUN GAO}

Department of Mathematics

Jinan University

Guangzhou, Guangdong 510632

P. R. China

e-mail: sunqian0112@foxmail.com

\begin{abstract}
In recent years, the Black-Scholes Option Model has been continuously improved and expanded, the main direction of the improvement is to add dividend payment or transaction cost to the classical option pricing formula, many researchers have improved the model to solve the use of partial differential equation model is more complex. In this paper, the Leland model and the traditional option pricing formula are combined with calculus, probability theory and mathematical statistics knowledge as well as the substitution of variables. At the same time, a modified formula of Black- Scholes model is given by using some conclusions of partial differential equation model. And our revised model is intuitive and easy to understand, simpler to compute. In this paper, combined with examples of comparative analysis, intuitively explain the transaction costs and continuous dividends on the impact of the option price.
\end{abstract}

2010 Mathematics Subject Classification: 97M30, 97M40.

Keywords and phrases: continuous dividend, transaction costs, Leland model, mathematical statistics.

Received September 30, 2017

(C) 2017 Scientific Advances Publishers 


\section{Introduction}

Option pricing is one of the core problems in financial mathematics. Option is a kind of derivative securities, and its value depends on the pricing of stock option stock option. Because the price change of stock is random, the price change of option is also random. In 1973, the American financial scientist Fisher Black and Myron Scholes deduced the differential equation of the European option price of the underlying asset for the non-dividend payment, that is, the Black-Scholes equation.

In recent years, scholars such as Xiaolei $\mathrm{Li}$ and Kaiding $\mathrm{Li}$ have considered the dividend payment on the basis of the classic Black-Scholes model, studied the European option pricing with dividend payment, Yiling $\mathrm{Wu}$ and Xiangxing Tao studied the option pricing formula with transaction cost and continuous dividend. Based on the traditional partial differential equation, Ming Chen also studied the option pricing model considering transaction fee and dividend payment. Their studies suggest that the classic Black-Scholes model did not take into account the transaction costs and dividends in the process of research will make the model in the real financial market in the practical decline, which also fully illustrates the Black-Scholes model of improved research is necessary.

We know that the classical option pricing formula has many kinds of derivation methods, binary tree model, continuous time model, partial differential equation model and so on. Many researchers in the study of the revised option pricing formula is also based on the classical option pricing formula derivation method, the majority of researchers in the Black-Scholes model based on the introduction of transaction costs and continuous dividends to study the method used is partial differential equation model, partial differential model is a very good way to derive, but the calculation is relatively cumbersome, in hence, we thought, could we develop simpler formulas for derivation? Thus, on the basis of the Leland model, we combine the calculus and probability theory and mathematical statistics knowledge and the substitution of variables, and also use the partial conclusion of the partial differential equation model 
to find the same modified option formula. Compared to the partial differential equation, our model is more intuitive and easier to understand, and the calculation becomes simpler.

The European option expiration date in the implementation of the features compared to American options easier to analyze, although most of the options trading in exchange for American options, but some properties of American options can be derived from the general nature of the European option, so this paper chooses European option as the research object.

\section{The Option Pricing Model for Payment of Transaction Fees and Dividend Payments}

The pricing formula for call options of classical Black-Scholes European:

$$
c(S, t)=S N\left(d_{1}\right)-K e^{-r(T-t)} N\left(d_{2}\right) .
$$

The pricing formula for put options:

$$
\begin{gathered}
p(S, t)=K e^{-r(T-t)} N\left(-d_{2}\right)-S N\left(-d_{1}\right), \\
d_{1}=\frac{\ln \left(\frac{S}{K}\right)+\left(r+\frac{\sigma^{2}}{2}\right)(T-t)}{\sigma \sqrt{T-t}}, d_{2}=d_{1}-\sigma \sqrt{T-t} .
\end{gathered}
$$

In Black-Scholes the classical pricing model, without considering the transaction costs, no transaction costs in the efficient market, investors can continue to change the risk through a combination of bond and stock options to hedge, usually assume that volatility is constant. But with the development and perfection of financial markets, because the price of the underlying assets is constantly changing, in order to avoid the risk of investors often have to adjust their positions for hedging continuously, there will be frequent trading, so using the Black-Scholes formula for option pricing in the actual operation, cannot completely ignore the transaction costs. 
The price of European-style bullish or put options for any rate-oftransaction fee is compared with the usual European option. The difference is that the volatility change is so that the option pricing model with the transaction fee is equivalent to the uncertainty option pricing model to discuss [1]. Within the period [0, T] considered, the range of $\sigma$ is $\left[\sigma_{\min }, \sigma_{\max }\right]$, where $\sigma_{\min }$ and $\sigma_{\max }$ is the given nonnegative constant.

The Leland model is introduced to deal with the volatility, the Leland number is $L e=\frac{k}{\sigma} \sqrt{\frac{8}{\pi \delta t}}$ defined, then the corrected volatility is [1]:

$$
\sigma_{L}^{2}= \begin{cases}\sigma^{2}(1-L e \operatorname{sgn}(\Gamma)) & \text { when investors are long } \\ \sigma^{2}(1+L e \operatorname{sgn}(\Gamma)) & \text { when investors are short }\end{cases}
$$

$L e=\frac{k}{\sigma} \sqrt{\frac{8}{\pi \delta t}}$ is the transaction cost parameter, $\sigma$ is volatility, $\delta t$ is the trading interval, and $k$ is a fixed rate of trading volume.

Suppose $\Gamma=\frac{\partial^{2} c}{\partial S^{2}}$, transaction costs depend on $\Gamma$, and $\Gamma$ is used to measure the degree of hedging in a portfolio, and for European call and put options without transaction costs, $\Gamma$ is always positive. When transaction costs exist, the symbol for $\Gamma$ is uncertain [2].

Let $\sigma_{\min }^{2}=\sigma^{2}(1-\alpha), \quad \sigma_{\max }^{2}=\sigma^{2}(1+\alpha)$.

The investors are long and short when they are:

$$
\begin{gathered}
\sigma_{L}^{2}= \begin{cases}\sigma_{\max }^{2}, & \Gamma<0, \\
\sigma_{\min }^{2}, & \Gamma>0,\end{cases} \\
\sigma_{L}^{2}= \begin{cases}\sigma_{\max }^{2}, & \Gamma>0, \\
\sigma_{\min }^{2}, & \Gamma<0 .\end{cases}
\end{gathered}
$$


Combined with the hypothesis of risk neutrality and no arbitrage in Leland (1985), the Black-Scholes partial differential equation of option price is obtained in the European call option pricing with uncertain volatility of transaction costs:

$$
r c=\frac{\partial c}{\partial t}+r S \frac{\partial c}{\partial S}+\frac{1}{2} \frac{\partial^{2} c}{\partial S^{2}} \sigma_{L}^{2} S^{2}
$$

If $L e>1$, then $\sigma_{L}^{2}$ is negative, the above equations are mathematically ill posed [3]. In order to change the problem of fixed solution into an adaptive problem, we must assume that $L e<1$. This shows that the transaction costs are relatively small or the risk of hedging cannot be too frequent, otherwise, the use of Leland model to ask for European options with transaction costs is not correct, extend it to the traditional model solution. We can see that $L e<1$.

Let $S$ be the spot stock price, $K$ is the option execution price, $r$ is the risk-free interest rate, $\sigma_{L}$ is the stock revised volatility, and $T$ is the expiration date [4].

For the European call options, the boundary conditions are: $c_{T}=\max \left(S_{T}-K, 0\right)$.

The volatility of the revised European call option:

$$
\sigma_{L}=\sigma^{2}\left(1+\frac{k}{\sigma} \sqrt{\frac{8}{\pi \delta t}}\right)=\sigma^{2}+2 k \sigma \sqrt{\frac{2}{\pi \delta t}} .
$$

For the European put option, its boundary condition is: $c_{T}=\max \left(K-S_{T}, 0\right)$.

The volatility of the revised European put option:

$$
\sigma_{L}=\sigma^{2}\left(1+\frac{k}{\sigma} \sqrt{\frac{8}{\pi \delta t}}\right)=\sigma^{2}+2 k \sigma \sqrt{\frac{2}{\pi \delta t}} .
$$

The option price is the present value of its future expected return, and the option is treated as a risk less asset under dynamic risk hedging, 
so we should discount the expected return on the option with the risk-free rate and get the expected return on the call for:

$$
c_{T}=e^{-r(T-t)} E\left[\max \left(S_{T}-K, 0\right)\right]
$$

The expected return of a put option is: $c_{T}=e^{-r(T-t)} E\left[\max \left(K-S_{T}, 0\right)\right]$.

In the reasoning of the classical Black-Scholes option pricing model, it is assumed that the underlying assets are paid without dividends, but in the real financial market, the stock investors often get a certain amount of dividends, and the dividends are paid in two cases. In the specified period of time to pay or in accordance with a certain percentage of continuous payment, the annual payment within a specified period of time can be paid on the dividend paid to each day, this can also be regarded as a dividend is continuous payment, so this article only discussed. In the case of continuous payments, the dividends paid on the ex-dividend date can be distributed to each day within a specified period of time, so that the dividends are considered to be continuous payments [5].

According to the Black-Scholes model is extended to continuous dividend payments, the stock and a similar stocks do not pay dividends compared to that in the risk neutral world, the total rate of return of stock must be $r$, and the dividend yield is $q$. So the dividend payment is equivalent to $S e^{q(T-t)}$ reduction in share prices, Therefore, the dividend paid $q$ stock price growth rate of $r-q$. If the stock price of the dividend is paid continuously, the price increases from $S$ at $t$ to $S_{T}$ at $T$. Then the stock price without red interest rates will increase from $S e^{-q(T-t)}$ at $t$ to $S_{T}$ at $T$. So that a European option based on a price of $S$, which pays a continuous red interest rate of $q$, can be of the same value as the corresponding European option based on a stock whose price is $S e^{-q(T-t)}$ that does not pay a dividend. Therefore, in order to price the European option based on the red interest rate, the current price of the stock can be 
reduced from $S$ to $S e^{-q(T-t)}$, using $S e^{-q(T-t)}$ instead of dividend paying European options in $S$ [6], [7].

The stock price process is derived from the knowledge of discrete models and has transaction costs and pays dividends:

$$
S_{T}=S e^{-q(T-t)} e^{r(T-t)-\frac{1}{2} \sigma_{L}^{2}(T-t)+\sigma_{L} \sqrt{T-t} z} .
$$

Among them, $q$ is the stock continuous dividend payment interest rate.

Put the stock price process into $c_{T}=e^{-r(T-t)} E\left[\max \left(S_{T}-K, 0\right)\right]$. We can see:

$$
c=e^{-r(T-t)} E\left[\max \left(S e^{(r-q)(T-t)-\frac{1}{2} \sigma_{L}^{2}(T-t)+\sigma_{L} \sqrt{T-t} z}-K, 0\right)\right] .
$$

\section{The Pricing Formula of Call Put Option is Derived for the Modified B-S Model}

We can extend the revised model to a similar stock without paying dividends, according to the formula of the Black-Scholes model to promote the transaction costs and the continuous dividend payments. The Black-Scholes model of the stock that does not pay dividends at the $T$ moment of the stock price $S_{T}$ this random variable is subject to a lognormal distribution, and the expected value of $S_{T}$ can be:

$$
E\left(S_{T}\right)=e^{r+\frac{1}{2} \sigma_{L}^{2}(T-t)}
$$

Therefore, the expected value of the dividend payment is: $E_{1}\left(S_{T}\right)=$ $e^{r-q+\frac{1}{2} \sigma_{L}^{2}(T-t)}$.

The expected value of the standard normal variable is: $E(z)=\int_{-\infty}^{\infty} x$ $\frac{1}{\sqrt{2 \pi}} e^{-\frac{x^{2}}{2}} d x$ 
Therefore, for the standard normal variable $z$, the present value expression of the expected value of the option can be written as:

$$
c=e^{-r(T-t)} \int_{-\infty}^{\infty}\left[\max \left(S e^{(r-q)(T-t)-\frac{1}{2} \sigma_{L}^{2}(T-t)+\sigma_{L} \sqrt{T-t} x}-K, 0\right)\right] \frac{1}{\sqrt{2 \pi}} e^{-\frac{x^{2}}{2}} d x
$$

Since the option value $c$ is positive, there must be

$$
S e^{(r-q)(T-t)-\frac{1}{2} \sigma_{L}{ }^{2}(T-t)+\sigma_{L} \sqrt{T-t} x}-K>0 .
$$

That is: $e^{(r-q)(T-t)-\frac{1}{2} \sigma_{L}^{2}(T-t)+\sigma_{L} \sqrt{T-t} x}>\frac{K}{S}$.

$$
\begin{gathered}
(r-q)(T-t)-\frac{1}{2} \sigma_{L}{ }^{2}(T-t)+\sigma_{L} \sqrt{T-t} x>\ln \left(\frac{K}{S}\right) \\
x>\frac{\ln \left(\frac{K}{S}\right)-\left(r-q-\frac{1}{2} \sigma_{L}{ }^{2}\right)(T-t)}{\sigma_{L} \sqrt{T-t}} .
\end{gathered}
$$

Let: $a=\frac{\ln \left(\frac{K}{S}\right)-\left(r-q-\frac{1}{2} \sigma_{L}^{2}\right)(T-t)}{\sigma_{L} \sqrt{T-t}}$.

Take $a$ as the lower bound of the integral.

Then $\quad c=e^{-r(T-t)} \int_{-\infty}^{\infty}\left[\max \left(S e^{(r-q)(T-t)-\frac{1}{2} \sigma_{L}^{2}(T-t)+\sigma_{L} \sqrt{T-t} x}-K, 0\right)\right]$ $\frac{1}{\sqrt{2 \pi}} e^{-\frac{x^{2}}{2}} d x \quad$ can $\quad$ be $\quad$ transformed into: $\quad c=\frac{1}{\sqrt{2 \pi}} \int_{a}^{\infty} e^{-r(T-t)}$ $\left(S e^{(r-q)(T-t)-\frac{1}{2} \sigma_{L}^{2}(T-t)+\sigma_{L} \sqrt{T-t} z}-K\right) e^{-\frac{x^{2}}{2}} d x$.

Divide the upper form into two integrals: 


$$
\begin{gathered}
I_{1}=\frac{1}{\sqrt{2 \pi}} \int_{a}^{\infty} e^{-q(T-t)} S e^{-\frac{1}{2} \sigma_{L}^{2}(T-t)+\sigma_{L} \sqrt{(T-t)} x} e^{-\frac{x^{2}}{2}} d x \\
I_{2}=-K e^{-r(T-t)} \frac{1}{\sqrt{2 \pi}} \int_{a}^{\infty} e^{-\frac{x^{2}}{2}} d x
\end{gathered}
$$

Simplify $I_{1}$ :

$$
\begin{aligned}
I_{1} & =\frac{1}{\sqrt{2 \pi}} \int_{a}^{\infty} e^{-q(T-t)} S e^{-\frac{1}{2} \sigma_{L}^{2}(T-t)+\sigma_{L} \sqrt{T-t} x} e^{-\frac{x^{2}}{2}} d x \\
& =\frac{1}{\sqrt{2 \pi}} \int_{a}^{\infty} e^{-q(T-t)} S e^{-\frac{1}{2}\left(x-\sigma_{L} \sqrt{T-t}\right)^{2}} d x .
\end{aligned}
$$

Variables are replaced by the upper form, let $y=x-\sigma_{L} \sqrt{T-t}$, therefore, $I_{1}$ becomes:

$$
\begin{aligned}
I_{1} & =\frac{1}{\sqrt{2 \pi}} \int_{a}^{\infty} e^{-q(T-t)} S e^{-\frac{1}{2}\left(x-\sigma_{L} \sqrt{T-t}\right)^{2}} d x \\
& =\frac{1}{\sqrt{2 \pi}} \int_{a-\sigma_{L} \sqrt{T-t}}^{\infty} e^{-q(T-t)} S e^{-\frac{y^{2}}{2}} d y .
\end{aligned}
$$

The symmetry of the standard normal distribution can be obtained:

$$
\begin{aligned}
I_{1} & =\frac{1}{\sqrt{2 \pi}} \int_{a-\sigma_{L} \sqrt{T-t}}^{\infty} e^{-q(T-t)} S e^{-\frac{y^{2}}{2}} d y \\
& =S e^{-q(T-t)} \frac{1}{\sqrt{2 \pi}} \int_{-\infty}^{-a+\sigma_{L} \sqrt{T-t}} e^{-\frac{y^{2}}{2}} d y .
\end{aligned}
$$

Let $d_{2}=-a$, then:

$$
I_{1}=S e^{-q(T-t)} \frac{1}{\sqrt{2 \pi}} \int_{-\infty}^{d_{2}+\sigma_{L} \sqrt{T-t}} e^{-\frac{y^{2}}{2}} d y
$$




$$
=S e^{-q(T-t)} N\left(d_{2}+\sigma_{L} \sqrt{T-t}\right) .
$$

By simplifying $I_{1}$, the symmetry of the standard normal distribution can be obtained:

$$
I_{2}=-K e^{-r(T-t)} \frac{1}{\sqrt{2 \pi}} \int_{a}^{\infty} e^{-\frac{x^{2}}{2}} d x=\frac{1}{\sqrt{2 \pi}}-K e^{-r(T-t)} \int_{-\infty}^{-a} e^{\frac{x^{2}}{2}} d x .
$$

Simplify $I_{2}: I_{2}=-K e^{-r(T-t)} N(-a)=-K e^{-r(T-t)} N\left(d_{2}\right)$.

Add $I_{1}$ and $I_{2}$ to get:

$$
c=S e^{-q(T-t)} N\left(d_{2}+\sigma_{L} \sqrt{T-t}\right)-K e^{-r \tau} N\left(d_{2}\right) .
$$

Among them: $d_{2}=-a=\frac{\ln \left(\frac{S}{K}\right)+\left(r-q-\frac{\sigma_{L}{ }^{2}}{2}\right)(T-t)}{\sigma_{L} \sqrt{T-t}}$.

Also because:

$$
d_{2}+\sigma_{L} \sqrt{T-t}=\frac{\ln \left(\frac{S}{K}\right)+\left(r-q-\frac{\sigma_{L}^{2}}{2}\right)(T-t)+\sigma_{L}^{2}(T-t)}{\sigma_{L} \sqrt{T-t}}=d_{1} .
$$

Therefore, $d_{2}=d_{1}-\sigma_{L} \sqrt{T-t}$.

So, we can get with the transaction costs and dividends to pay the European call options formula:

$$
\begin{gathered}
c=S e^{-q(T-t)} N\left(d_{1}\right)-K e^{-r(T-t)} N\left(d_{2}\right), \\
d_{1}=\frac{\ln \left(\frac{S}{K}\right)+\left(r-q+\frac{\sigma_{L}{ }^{2}}{2}\right)(T-t)+\sigma_{L}^{2}(T-t)}{\sigma_{L} \sqrt{T-t}}, d_{2}=d_{1}-\sigma_{L} \sqrt{T-t}, \\
\sigma_{L}^{2}=\sigma^{2}+2 k \sigma \sqrt{\frac{2}{\pi \delta t}} .
\end{gathered}
$$


Bullish put options parity formula:

$$
c(S, t)+K e^{-r(T-t)}=p(S, t)+S e^{-q(T-t)} .
$$

According to the bullish and bullish option pricing formula, the pricing formula of the put option can be obtained. The put option needs to change the volatility $\sigma_{L}^{2}=\sigma^{2}+2 k \sigma \sqrt{\frac{2}{\pi \delta t}}$ to $\sigma_{L}^{2}=\sigma^{2}-2 k \sigma \sqrt{\frac{2}{\pi \delta t}}$, [6].

As a result, the pricing formula of European put options with transaction costs and dividend payments is derived:

$$
\begin{gathered}
p=K e^{-r(T-t)} N\left(-d_{2}\right)-S e^{-q(T-t)} N\left(-d_{1}\right), \\
d_{1}=\frac{\ln \left(\frac{S}{K}\right)+\left(r-q+\frac{\sigma_{L}{ }^{2}}{2}\right)(T-t)+\sigma_{L}{ }^{2}(T-t)}{\sigma_{L} \sqrt{T-t}}, d_{2}=d_{1}-\sigma_{L} \sqrt{T-t} \\
\sigma_{L}^{2}=\sigma^{2}-2 k \sigma \sqrt{\frac{2}{\pi \delta t}} .
\end{gathered}
$$

\section{Research on the Application of Fixed Option Pricing Formula}

\section{Give an example:}

Consider the S \& P 500 European call option, six months expiring, the present value of the index is 930 , the implementation price is 900 , the risk-free rate is $8 \%$ per year, the index volatility is $20 \%$ per year, the sixmonth expectation, the total dividend yield is $1.5 \%$ and the transaction fee is 0.003 .

In this example, $S=930, K=900, r=0.08, \sigma=0.2, \tau=6 / 12$, $q=1.5 \% \times 12 / 6=3 \%, k=0.003$, let $\delta t=0.5$.

An option price without dividend payments and transaction costs, whose Black-Scholes formula is: 


$$
\begin{gathered}
c(S, t)=S N\left(d_{1}\right)-K e^{-r(T-t)} N\left(d_{2}\right), \\
d_{1}=\frac{\ln \left(\frac{S}{K}\right)+\left(r+\frac{\sigma^{2}}{2}\right)(T-t)}{\sigma \sqrt{T-t}}, d_{2}=d_{1}-\sigma \sqrt{T-t} .
\end{gathered}
$$

The solution can be obtained by Matlab: $c(S, t)=89.7724$.

When there is no transaction cost, the option pricing formula for dividend payment is:

$$
\begin{gathered}
c(S, t)=S e^{-q(T-t)} N\left(d_{1}\right)-K e^{-r(T-t)} N\left(d_{2}\right), \\
d_{1}=\frac{\ln \left(\frac{S}{K}\right)+\left(r-q+\frac{\sigma^{2}}{2}\right)(T-t)}{\sigma \sqrt{T-t}}, d_{2}=d_{1}-\sigma \sqrt{T-t} .
\end{gathered}
$$

The solution can be obtained by Matlab: $c(S, t)=80.0425$.

Option pricing formula for no payment of dividends with transaction costs:

$$
\begin{gathered}
c(S, t)=S N\left(d_{1}\right)-K e^{-r(T-t)} N\left(d_{2}\right) \\
d_{1}=\frac{\ln \left(\frac{S}{K}\right)+\left(r+\frac{\sigma_{L}^{2}}{2}\right)(T-t)}{\sigma_{L} \sqrt{T-t}}, d_{2}=d_{1}-\sigma_{L} \sqrt{T-t}, \sigma_{L}^{2}=\sigma^{2}+2 k \sigma \sqrt{\frac{2}{\pi \delta t}} .
\end{gathered}
$$

The solution can be obtained by Matlab: $c(S, t)=90.5255$.

Option pricing formula with transaction costs and continuous dividend payments:

$$
\begin{gathered}
c(S, t)=S e^{-q(T-t)} N\left(d_{1}\right)-K e^{-r(T-t)} N\left(d_{2}\right), \\
d_{1}=\frac{\ln \left(\frac{S}{K}\right)+\left(r-q+\frac{\sigma_{L}{ }^{2}}{2}\right)(T-t)}{\sigma_{L} \sqrt{T-t}}, d_{2}=d_{1}-\sigma_{L} \sqrt{T-t}, \sigma_{L}^{2}=\sigma^{2}+2 k \sigma \sqrt{\frac{2}{\pi \delta t}} .
\end{gathered}
$$


The solution can be obtained by Matlab: $c(S, t)=80.8269$.

In the model with transaction costs, taking $\delta t$ is 0.5 , and does not represent all the interval time, and $\delta t$ is further subdivided into solutions, let

$$
\begin{aligned}
\delta t= & 0.025,0.050,0.075,0.100,0.125,0.150,0.175,0.200,0.225,0.250 \\
& 0.275,0.300,0.325,0.350,0.375,0.400,0.425,0.450,0.475,0.500
\end{aligned}
$$

An option pricing formula with dividend payments and transaction costs is derived. The results are as follows:

Table 1. Volatility and option price when $\delta t$ is not corrected at the same time

\begin{tabular}{|c|c|c|c|c|c|}
\hline$\delta t$ & 0.025 & 0.050 & 0.075 & 0.100 & 0.125 \\
\hline$\sigma_{L}$ & 0.2146 & 0.2104 & 0.2086 & 0.2074 & 0.2067 \\
\hline$C(S, t)$ & 83.4247 & 82.4481 & 82.0305 & 81.7523 & 81.5901 \\
\hline$\delta t$ & 0.150 & 0.175 & 0.200 & 0.225 & 0.250 \\
\hline$\sigma_{L}$ & 0.2061 & 0.2056 & 0.2053 & 0.2050 & 0.2047 \\
\hline$C(S, t)$ & 81.4512 & 81.3355 & 81.2661 & 81.1967 & 81.1273 \\
\hline$\delta t$ & 0.275 & 0.300 & 0.325 & 0.350 & 0.375 \\
\hline$\sigma_{L}$ & 0.2045 & 0.2043 & 0.2042 & 0.2040 & 0.2039 \\
\hline$C(S, t)$ & 81.0811 & 81.0348 & 81.0117 & 80.9655 & 80.9424 \\
\hline$\delta t$ & 0.400 & 0.425 & 0.450 & 0.475 & 0.500 \\
\hline$\sigma_{L}$ & 0.2037 & 0.2036 & 0.2035 & 0.2034 & 0.2034 \\
\hline$C(S, t)$ & 80.8962 & 80.8731 & 80.8500 & 80.8269 & 80.8269 \\
\hline
\end{tabular}




\section{Result Analysis}

Table 2. Comparison of prices before and after the Black-Scholes model revision

\begin{tabular}{|c|c|c|c|c|}
\hline & $\begin{array}{c}\text { No dividend } \\
\text { payment } \\
\text { No transaction } \\
\text { cost }\end{array}$ & $\begin{array}{c}\text { Dividend } \\
\text { payment } \\
\text { No transaction } \\
\text { cost }\end{array}$ & $\begin{array}{c}\text { No dividend } \\
\text { payment } \\
\text { Transaction cost }\end{array}$ & $\begin{array}{c}\text { Dividend } \\
\text { payment } \\
\text { Transaction cost }\end{array}$ \\
\hline$C(S, t)$ & 89.7724 & 80.0425 & 90.5255 & 80.8269 \\
\hline
\end{tabular}

According to the above empirical analysis, the revised option price and the classic Black-Scholes pricing model to compare the results can be drawn as follows:

There is a continuous dividend payment when the option price is lower than the theory, in the case of no transaction costs, there are bonus payments compared to the price of non-dividend payment decreased by $10.8384 \%$, in the case of transaction costs, there are bonus payments compared to the price of non-dividend payment decreased by $10.7137 \%$, indicating that there are dividends to pay the price of options have a certain impact.

There is a transaction cost when the option price is higher than the theoretical price, there is no dividend payment in the case, there is no transaction costs compared to the transaction price increase of $0.8389 \%$, in the case of a dividend payment, there is no transaction costs compared to the transaction price increases by $0.9800 \%$, indicating that the effect of transaction fees on option pricing is very small.

The volatility of $\delta t$ obtained at Table 1 is not corrected at the same time as the option price. The result is drawn in Matlab: 


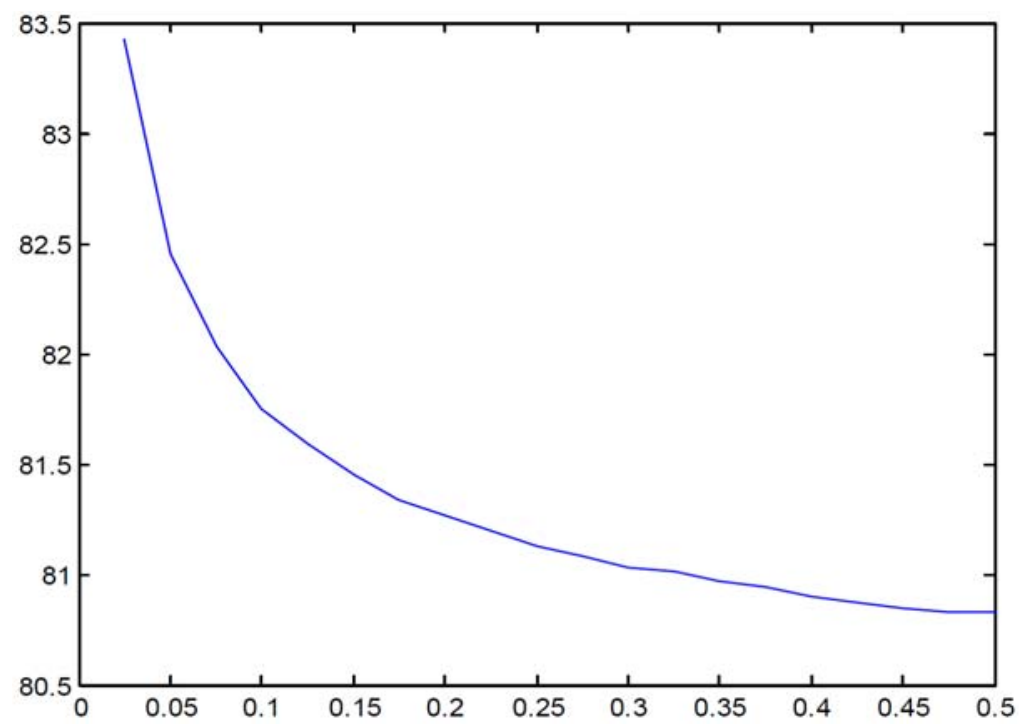

Figure 1. The price of the option when $\delta t$ is not modified at the same time.

As can be seen from Figure 1, with the increase of $\delta t$, transaction costs gradually decrease, and with the increase of interval $\delta t$, transaction costs change more and more small. In the financial sense, the more frequent the adjustment ( $\delta t$ is smaller), the higher the transaction costs, so the seller of the option charges the price of the option more [9].

\section{Conclusion}

The dividend factor is generally applicable to stock options. In the absence of other factors, the dividend will be equal to the price before the ex-dividend date minus the dividend, and the dividend will lower the stock price. According to the effect of the stock price on the option price, the decrease in the stock price will cause the call price to fall and the price of the put option will rise. Moreover, call option holders although buy stock rights, but before the real stock, is not eligible to participate in dividends, and although the option holder can sell the stock, but before 
the actual implementation period, does not lose in the dividend distribution of qualification to price and the expected dividend option of the size of the moves in the opposite direction however, price and the expected dividend option of the size of the positive changes.

At present, Chinese warrants market, the transaction costs are generally three thousandths, if the big customers and brokerage agreement down commission, then the transaction costs will be lower, especially in Chinese stock market has not yet short of the case, not unlimited hedging, Le $<1$ manifest that the transaction fee is relatively small or hedge the risk of the process cannot be too frequent, it is just in line with our conclusions. So in terms of unilateral transactions, transaction costs are usually negligible [10].

The conclusion of the above case is consistent with the actual operation of the market, indicating that the revised option formula has a certain reference value to the financial market, but not exactly the same as the actual market, the option pricing formula there are many need to study place, how to use the simple method on the basis of the existing method to improve is also a very necessary problem.

\section{References}

[1] Zhengjie Li, Xingyong Zhang and Yan Liang, Pricing of European put options with uncertain volatility of payment transactions, Journal of Xuzhou Normal University 6-28(2) (2010), 35-38.

[2] Yuquan Guo, The Mathematical Model of Financial Derivatives, Beijing: Science Press, 2012.

[3] John C. Hull, Options Futures and Other Derivatives, Beijing People's Posts and Telecommunications Press (2013), 12.

[4] Yabin Du, Financial Modeling, Peking University Press (2015), 6.

[5] Yiling $\mathrm{Wu}$ and Xiangxing Tao, Option pricing formula with transaction cost and continuous dividend, Journal of Ningbo University 6, 22(2) (2009), 230-234.

[6] Xiaolei Li and Kaiding Li, Mathematical model of financial derivatives, Journal of Applied Mathematics 20 (2007), 102-104. 
[7] Xiaoying Zheng and Jinxian Chen, Option pricing research with transaction costs, Journal of University of Electronic Science and Technology of China 2(2) (2000), 110-102.

[8] Joseph Stampfli and Victor Goodman, Financial Mathematics, Beijing: Mechanical Industry Press, (2004), 3.

[9] Yong Tang and Jixiang Chen, Experimental study on the risk avoidance strategy of transaction costs, Shanghai Management Science 6, 31(3) (2009), 24-26.

[10] Ming Chen, Black-Scholes option pricing correction model and its application, Statistics and Decision 7(331) (2011), 135-137. 